\title{
ON THE ORDERED SET OF REFLECTIVE SUBCATEGORIES
}

\author{
G.M. KELLY
}

Given a category $A$, we consider the (often large) set Ref $A$ of its reflective (full, replete) subcategories, ordered by inclusion. It is known that, even when $A$ is complete and cocomplete, wellpowered and cowellpowered, the intersection of two reflective subcategories need not be reflective. Supposing that $A$ admits (i) small limits and (ii) arbitrary (even large) intersections of strong subobjects, we prove that an infimum $\wedge_{i} C_{i}$ in Ref A must necessarily be the intersection $\cap_{i} \mathcal{C}_{i}$. Accordingly Ref A is not in general, even for good $A$, a complete lattice. We show, however, under the same conditions on $A$, that Ref $A$ does admit small suprema $V_{i} C_{i}$, given by the closure in $A$ of the union $U_{i} C_{i}$ under the limits of types (i) and (ii) above.

\section{Strongly complete categories}

We suppose an inaccessible cardinal $\infty$ chosen once for all, and call a set small if its cardinal is less than $\infty$. The morphisms of any category $A$ form a set, and $A$ is small if this set is small; while $A$

Received 28 August 1986. The author gratefully acknowledges the assistance of the Australian Research Grants Scheme.

Copyright clearance Centre, Inc. Serial-fee code: 0004-9727/87 $\$ A 2.00+0.00$. 
is locally small if each hom-set $A(A, B)$ is small. We use large to mean not small. Although a category is said to be complete when it admits all small limits, an ordered set is called a complete lattice only when it admits alZ suprema and infima - even large ones if it is a large set.

We refer to [7] for the definition and properties of strong monomorphisms. We call a category strongly complete if, besides admitting small limits, it admits the fibred product (or intersection) of any family $\left(f_{\alpha}: A_{\alpha} \rightarrow B\right)$ of strong monomorphisms; in other words, it admits all intersections of strong subobjects. Of course a category is strongly complete if it is complete and wellpowered, or even weakly wellpowered in the sense that each object has but a small set of strong subobjects.

In a strongly-complete category, the epimorphisms and the strong monomorphisms form a factorization system: given $f: A \rightarrow B$ we take for $i: C \rightarrow B$ the intersection of those strong subobjects of $B$ through which $f$ factorizes then $f=i p$, and $p$ is epimorphic by Lemma 4.4 of [7].

We use subcategory to mean full replete subcategory. A subcategory $B$ of the strongly-complete $A$ is said to be closed in $A$ under strong Zimits if the limit in $A$ of a diagram in $B$ lies in $B$, whenever the diagram is either small or else of the form $\left(f_{\alpha}: A_{\alpha} \rightarrow B\right)$ where the maps $f_{\alpha}$ in $B$ are strong monomorphisms in A. Clearly the intersection of subcategories closed under strong limits is itself so closed. Given any subcategory $D$ of $A$, there is accordingly a smallest subcategory $D^{*}$ containing $D$ which is closed in $A$ under strong limits; we call $D^{*}$ the strong-limit closure of $D$ in $A$.

PROPOSITION 1. If $B$ is closed in the strongly-complete $A$ under strong limits, every strong monomorphism $f: C \rightarrow B$ in $B$ is a strong monomorphism in A. Accordingly $B$ is itself strongly complete, and the inclusion $B \rightarrow A$ preserves small limits and arbitrary intersections of strong monomorphisms.

Proof. Consider the totality of those maps $j: E_{j} \rightarrow B$ through which $f$ factorizes and which are strong monomorphisms in $A$ with $E_{j} \in B$. Then their intersection $k: E \rightarrow B$ in $A$ is a strong 
monomorphism in $A$, and $E \in B$ since $B$ is closed in $A$ under strong limits. Let the factorization of $f$ through $k$ be $f=k p$; we show that $p$ is invertible, so that $f$ like $k$ is a strong monomorphism in A . Since $f$ is by hypothesis a strong monomorphism in $B$, so is $p$; thus it suffices to show that $p$ is epimorphic in $B$. Let $x, y: E \rightarrow F$ in $B$ satisfy $x p=y p$, and let $z: G \rightarrow E$ be the equalizer in $A$ of $x$ and $y$. Since $B$ is closed in $A$ under small limits, we have $G \in B$; and $f$ factorizes through $k z$, which is a strong monomorphism in $A$. By the definition of $k$, therefore, $z$ is invertible and $x=y$.

\section{Strong cogenerators}

The definition in [7] of strong monomorphism admits an evident generalization to a definition of a strongly monomorphic family $\left(f_{\alpha}: A \rightarrow B_{\alpha}\right)$, in such a way that, if the product $\pi B_{\alpha}$ exists, the family is strongly monomorphic if and only if the corresponding map $A \rightarrow \Pi B{ }_{\alpha}$ is so.

A small set $G$ of objects of $A$ is said to be a strong cogenerator if, for each $A \in A$, the family $\left(h: A \rightarrow G_{h}\right)$ of all maps with codomain in $G$ is strongly monomorphic. When $A$ is complete and locally small, this is to say that the canonical map $\Pi_{A}: A \rightarrow \Pi_{G \in G}\{A(A, G) \pitchfork G\}$ (where $X \pitchfork G$ denotes the product of $X$ copies of $G$ ) is a strong monomorphism. From the form of the Special Adjoint Functor Theorem given by Börger et al. in Corollary 1.10 of [3], it follows that:

PROPOSITION 2. Let the strongly-complete and locally-small A have a strong cogenerator, and let $B$ be locally small. Then a fionctor $U: A \rightarrow B$ has a left adjoint if and only if it preserves small limits (and hence monomorphisms), and sends an intersection of strong monomorphisms to an intersection of monomorphisms.

If $G$ is a strong cogenerator, the set-valued functors $A(-, G)$ for $G \in G$ jointly reflect isomorphisms. Taking for simplicity the case of a locally-small complete $A$, suppose $f: A \rightarrow B$ to be such that each $A(f, G)$ is invertible. The naturality of $\eta$ gives $u n_{A}=\eta_{B} f$ where $u$ 
is the invertible map $\pi_{G}\{A(f, G) \pitchfork G\} ;$ since $u \eta_{A}$ is a strong monomoxphism, so is $f$. On the other hand, $G$ being a fortiori a cogenerator since the $n_{A}$ are a fortiori monomorphic, the $A(-, G)$ are jointly faithful and hence jointly reflect epimorphisms, so that $f$ is epimorphic and hence invertible.

Suppose conversely that the small set $G$ is such that the $A(-, G)$ jointly reflect isomorphims. Then if $\eta_{A}$ factorizes through an epimorphism $f: A \rightarrow B$, this $f$ is invertible. For to say that $\eta_{A}$ factorizes through $f$ is to say that every $h: A \rightarrow G$ with $G \in G$ factorizes through $f$, or that each $A(f, G)$ is surjective; but $A(f, G)$ is injective since $f$ is epimorphic.

If $A$ is such that $g: A \rightarrow C$ is a strong monomorphism whenever $g$ factorizes through no non-trivial epimorphism $f: A \rightarrow B$ (which is the case whenever $A$ admits finite colimits, or whenever epimorphisms and strong monomorphisms constitute a factorization system on $A$, , a small set $G$ is a strong cogenerator exactly when the $A(-, G)$ for $G \in G$ jointly reflect isomorphisms; this is really a special case of Proposition 4.3 of Im and Kelly [6]. Since we have seen that epimorphisms and strong monomorphisms do form a factorization system when $A$ is strongly complete, we have:

PROPOSITION 3. A smaZl set $G$ of objects of a locally-small and strongly-complete A form a strong cogenerator if and only if a mop $f$ is invertible whenever $A(f, G)$ is so for each $G \in G$.

PROPOSITION 4. Let $D$ be a small subcategory of the locally-small and strongly-complete $A$, and let $D^{*}$ be the strong-limit closure of $D$ in $A$. Then $D$ is a strong cogenerator for $D^{*}$ (which is itself strongly complete by Proposition 1.)

Proof. By Proposition 3, we have to show that a map $f$ in $D$ is invertible if $A(f, D)$ is so for each $D \in D$. Consider the subcategory $B$ of $A$ given by all those objects $B$ of $A$ for which $A(f, B)$ is invertible. If any diagram in $B$ admits a limit in $A$, this limit lies 
in $B$; for $A\left(f, \lim B_{i}\right) \cong \lim A\left(f, B_{i}\right)$. So $B$ is closed in $A$ under strong limits; therefore, since it contains $D$, it contains $D^{*}$. Thus $f$ is invertible since it is a map in $D^{*}$ with $A(f, B)$ invertible for each $B \in D^{*}$.

Propositions 1, 2, and 4 now give:

PROPOSITION 5. If $D$ is a small subcategory of the locally-small and strongly-complete $A$ and $D^{*}$ is the strong-limit closure of $D$ in $A$, then $D^{*}$ is reflective in $A$.

\section{Infima of reflective subcategories}

We write $\operatorname{Ref} \Lambda$ for the set of reflective subcategories of $A$, ordered by inclusion. We use $V$ and $\wedge$ to denote suprema and infima in Ref $A$, so far as they exist, retaining $U$ and $\cap$ for union and intersection.

Remark 6. Even when A is locally small, complete and cocomplete, wellpowered and cowellpowered, and has a generator and a strong cogenerator, the intersection of two reflective subcategories need not be reflective. The following simple counter-example is contained in a forthcoming article [1] by Adamek and Rosicky. An object of $A$ is a set with two topologies, a first and a second; and a map $f: A \rightarrow B$ is a function continuous both for the first topology and for the second. The subcategory $C_{1}$ [respectively $C_{2}$ ] consists of those objects for which the first [respectively second] topology is compact Hausdorff. A reflexion of $A \in A$ into $C_{1}$ is given by taking its stone-Čech compactification $r: A \rightarrow B$ with respect to the first topology, and giving to $B$ as its second topology the final one with respect to $r$; similarly for the reflexion into $\mathcal{C}_{2}$. Yet $\mathcal{C}_{1} \cap \mathcal{C}_{2}$ is not reflective; the formal proof is in [1], but the intuition is clear enough - if we take the reflexion of $A$ into $C_{1}$, and then the reflexion of this into $C_{2}$, and then the reflexion of this last into $\mathcal{C}_{1}$, and so on alternately, the cardinal of the successive reflexions increases unboundedly. (The author knows of no 
such counter-example for a well-behaved A with a strong generator.\}

This counter-example does not of itself show that $\operatorname{Ref} A$ for such an $A$ may lack binary infima; but it does so when combined with the following:

THEOREM 7. Let $A$ be Zocally small and strongly complete. If a subset $\left\{C_{i}\right\}$ of $\operatorname{Ref} A$ admits an infimon $\wedge C_{i}$, this must be the intersection $\cap \mathrm{C}_{i}$.

Proof. If cony diagram in a reflective $C_{i}$ admits a limit in $A$, this limit lies in $C_{i}$; hence each $C_{i}$ is closed in $A$ under strong limits. Thus if $D \in \cap \mathcal{C}_{i}$ and if $\{D\}$ denotes the subcategory of $A$ consisting of $D$ alone, its strong-limit closure $\{D\}^{*}$ is contained in $\cap C_{i}$. Since $\{D\}^{*}$ is reflective in $A$ by Proposition 5, we have $\{D\} * \subset \wedge C_{i}$. Since this is true for every $D \in \cap C_{i}$ and since $D \in\{D\}^{*}$, we have $\cap \mathcal{C}_{i} \subset \wedge C_{i}$; whence $\cap C_{i}=\wedge c_{i}$.

Remark 8. Since Ref A for a strongly-complete A, or even for an A so good as that of Remark 6, may lack binary infima, it is not in general a complete lattice. Yet we show in Theorem 14 below that Ref $A$ admits small suprema when $A$ is strongly complete. It follows that Ref $A$ is a large set for the $A$ of Remark 6 . Since this $A$ has a strong cogenerator $G=\left\{G_{1}, G_{2}, G_{3}\right\}$, where each $G_{i}$ is a two-element set and the respective pairs of topologies are (chaotic, chaotic), (chaotic, Sierpinski), and (Sierpinski, chaotic), Ref A can be large even when the locally-small strongly-complete $A$ has a strong cogenerator. The following result, therefore, has no converse:

PROPOSITION 9. Let A be locally small and strongly complete. If Ref A is a small set, A has a strong cogenerator.

Proof. Since $\{D\}^{*}$ is reflective in $A$ for each $D \in A$ by Proposition 5, the set of distinct $\{D\}^{*}$ is small; let it consist of the $\{G\}^{*}$ where $G$ runs through the small set $G$. Given $A \in A$, let 
$\{A\}^{*}=\{H\}^{*}$ where $H \in G$. By Proposition 4, $H$ is a strong cogenerator for $\{A\}^{*}$, so that the canonical $\zeta: A \rightarrow A(A, B)$ if $B$ is a strong monomorphism in $\{A\}^{*}$. Here $A(A, H) \pitchfork E$ is in the first instance the product in $\{A\}^{*}$; but it is equally the product in $A$. Moreover $\zeta$ is a strong monomorphism in $A$ by Proposition 1 . Since $\zeta$ factorizes through $\eta_{A}: A \rightarrow \Pi_{G \in G}\{A(A, G) \pitchfork G\}$, the latter too is a strong monomorphism in $A$; so that $G$ is a strong cogenerator for $A$.

\section{Reflective factorization systems}

We call a set $M$ of maps in a category $A$ a skein if it contains the isomorphisms and is closed under composition. A skein $M$ is stable under pulzbacks if the pullback of a map in $M$ along any map in $A$ is again in $M$. The skein $M$ is stable under fibred products if, whenever a family $\left(f_{\alpha}: A_{\alpha} \rightarrow B\right)$ of maps in $M$ admits a fibred product $h: C \rightarrow B$, we have $h \in M_{i}$ similarly for stability under small or finite fibred products. (Note the distinction between stability under pullbacks and stability under binary fibred products.) Stability under the intersections of strong monomorphisms means stability under those fibred products, possibly large, in which each $f_{\alpha}$ is a strong monomorphism.

For maps $e$ and $m$ in $A$ we write $e \downarrow m$ if, for every commutative square $v e=m u$, there is a unique "diagonal" $w$ with $w e=u$ and $m w=v$. If $N$ is any set of maps in $A$ we write $N^{+}=\{m \mid n+m$ for all $n \in N\}$ and $N^{\uparrow}=\{e \mid e+n$ for all $n \in N\}$. By Proposition 2.1.1 of Freyd and Kelly [5], $N^{\downarrow}$ is a skein, stable under pullbacks and fibred products, such that

$$
\text { if } f g \text { and } f \text { are in } N^{\downarrow} \text {, so is } g \text {. }
$$

We recall from [5] that a factorization system $(E, M)$ on $A$ consists of two skeins $E$ and $M$ such that every map $f$ in $A$ admits a factorization $f=m e$ with $m \in M$ and $e \in E$, and such that $e+m$ for each $e \in E$ and $m \in M$. This last requirement may be expressed equivalently as $M \subset E^{+}$, or as $E \subset M^{+}$; in fact, by Proposition 2.2.1 
of [5], every factorization system satisfies $M=E^{\dagger}$ and $E=M^{\uparrow}$, and hence is fully determined by the knowledge of either $E$ or $M$. In any factorization system $E \cap M$ consists of the isomorphisms, since $e \downarrow e$ only for invertible $e$. We order factorization systems by setting $(E, M) \leq\left(E^{\prime}, M^{\prime}\right)$ when $M \subset M^{\prime}$; or equivalently when $E^{\prime} \subset E$.

By the dual of (1), any factorization system $(E, M)$ satisfies

$$
\text { if } f g \text { and } g \text { are in } E \text {, so is } f \text {; }
$$

we call ( $E, M)$ a reflective factorization system if it also satisfies

$$
\text { if } f g \text { and } f \text { are in } E \text {, so is } g \text {. }
$$

It was proved by Cassidy, Hébert, and Kelly in Section 2 of [4] that, when $A$ is strongly finitely complete (that is, when $A$ admits finite limits and arbitrary intersections of strong monomorphisms), there is an order-preserving bijection between reflective factorization systems on $A$ and reflective subcategories of $A$. We now give a modification of their proof of that result; the new proof provides extra information on $M$ needed in our applications below.

PROPOSITION 10. Let $C$ be a reflective subcategory of the stronglyfinitely-complete $A$, the reflexion of $A$ into $C$ being $\rho_{A}: A \rightarrow r A$. Let $\Phi C=(E, M)$ where $E$ is the set of maps inverted by $r: A \rightarrow A$ and where $M$ is the smallest skein in $A$ containing mor $C$ and stable under pullbacks and arbitrary intersections of strong monomorphisms. Then

$$
C=E^{\perp}={ }_{D f}\{A \in A \mid A(e, A) \text { is invertible for alz } e \in E\} \text {, }
$$

and $(E, M)$ is a reflective factorization system with factorizations constructed as in (5) and (6) below.

Proof. If $C \in C$ and $e \in E$, we have $A(e, C)$ invertible since it is conjugate to the invertible $\mathcal{C}(r(e), C)$ : thus $C \subset E^{\perp}$. We may always so choose the reflexion that $\rho_{C}: C \rightarrow r C$ for $C \in C$ is $1: C \rightarrow C$; then $r\left(\rho_{A}\right)=1$ for any. $A \in A$. If $A \in E^{\perp}$ we have $A\left(\rho_{A}, A\right)$ invertible, 


\section{Reflective subcategories}

since $\rho_{A} \in E$; so there is an $h: r A+A$ with $h \rho_{A}=1$, and now $\rho_{A} \rho_{A}=\rho_{A}$ gives $\rho_{A} h=1: r A \rightarrow r A ;$ thus $\rho_{A}$ is invertible and $A \in C$. This proves (4).

$E$ is clearly a skein, and $M$ is a skein by definition. For $C \in C$, we have $C \in E^{\perp}$ by (4); this is equally the assertion that $C \rightarrow 1$, where 1 is the terminal object of $A$, lies in $E^{\downarrow}$. It follows from (1) that mor $C \subset E^{\dagger}$. Since the skein $E^{\downarrow}$, by the remarks preceding (1), is stable under pullbacks and fibred products, it follows from the definition of $M$ that $M \subset E^{\downarrow}$.

\section{Since $E$ clearly satisfies (3), it remains to show that any} $f: A \rightarrow B$ in $A$ has an (E,M) factorization. In the diagram

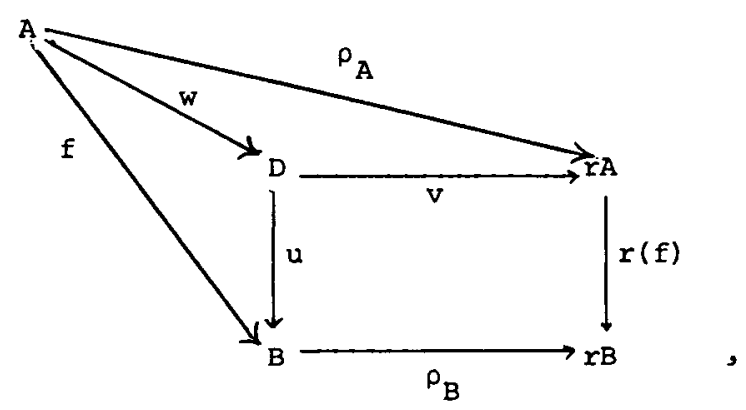

let the square be a pullback. Since $r(f) \in \operatorname{mor} C \subset M$, we have $u \in M$ by the definition of $M$. Now let $k: E \rightarrow D$ be the intersection of all those strong subobjects of $D$, lying in $M$, through which $w$ factorizes; then we have a factorization

$$
A \underset{\omega}{\longrightarrow} D=A \underset{e}{\longrightarrow} E \underset{k}{\longrightarrow} D
$$

where the strong monomorphism $k$ lies in $M$ by the definition of $M$. We now show that $e \in E$, so that $f$ has (uk)e as its $|E, M|$ factorization. 
Since $r\left(\rho_{A}\right)=1$, applying $r$ to the top triangle of (5) shows that $r(w)$ is a coretraction; whence $r(e)$ is a coretraction by (6). Let the analogue of (5) with $e$ in place of $f$ be

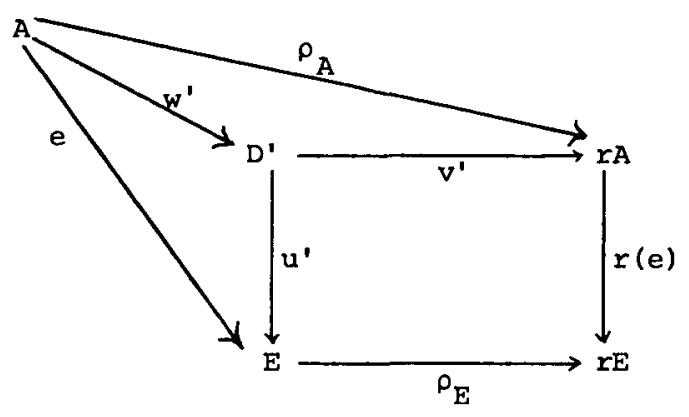

Since $r(e)$, being a coretraction, is a strong monomorphism, so is its pullback $u^{\prime}$. Being a strong monomorphism in $M$ through which $e$ factorizes, $u^{\prime}$ is invertible by the definition of $k$; so we may as well take $D^{\prime}=E, u^{\prime}=1$, and $w^{\prime}=e$. Applying $r$ to the top triangle of (7) gives $r\left(v^{\prime}\right) r(e)=1$, while applying $r$ to the square in (7) gives $r(e) r\left(v^{\prime}\right)=1$. So $r(e)$ is invertible and $e \in E$.

PROPOSITION 11. For any factorization system $(E, M)$ on an $A$ with a terminal object 1 , define a subcategory $C=\Psi(E, M)$ by

$$
C=M 1={ }_{D f}\{A \in A \mid A+1 \text { Zies in } M\} .
$$

Then $\mathrm{C}$ is reflective in $\mathrm{A}$, the reflexion $\rho_{A}: A \rightarrow r A$ being the E-part of the $(E, M)$ factorization of $A \rightarrow 1$.

THEOREM 12. FOr a strongly-finitely-complete $A$, the ciearly order-preservingl functions $\Phi$ and $\Psi$ constitute a bijection between reflective factorization systems on A and reflective subcategories of A.

Proof. $\Psi \Phi C=M / 1$, where $M$ is as in Proposition 10. Since $M$ is $E^{+}$, we have $M / 1=E^{\perp}$, which is $C$ by (4); hence $\Psi \Phi=1$. 
Let $\Psi(E, M)$, where $(E, M)$ is now reflective, be $\mathcal{C}$ as in Proposition 11, and let $\Phi C$ be $\left(E^{\prime}, M^{\prime}\right)$. Then $r(f)$ for $f: A \rightarrow B$ in $A$ is the unique map rendering commutative

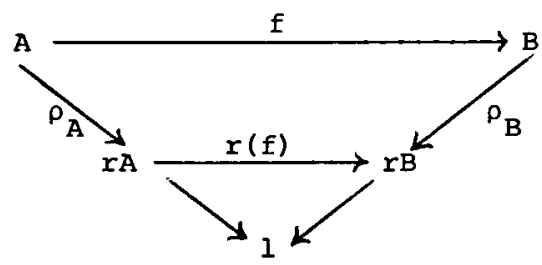

Since $r A \rightarrow 1$ and $r B \rightarrow 1$ lie in $M$, we have $r(f) \in M$ by (1). Hence $r(f)$ is invertible, so that $f \in E^{\prime}$, if and only if $r(f) \in E$. Since $\rho_{A} \in E$, this is equivalent by (2) to $r(f) \rho_{A} \in E$, and hence to $\rho_{B} f \in E$. since $\rho_{B} \in E$, this last is equivalent by (3) to $f \in E$. so $E^{\prime}=E$, and $\Phi \Psi=1$.

The following is contained in Borceux and Kelly [2] in the special case in which $\mathcal{C}$ is a localization, in the sense that the reflexion $r$ is left exact:

PROPOSITION 13. Let the reflective subcategory $C$ of the stronglyfinitely-complete $A$ correspond as in Theorem 12 to the reflective factoriation system $(E, M)$. Then if $g f \in E$ and $f \in M$, the map $f$ is a strong monomorphism.

Proof. Since $r(g f)=r(g) r(f)$ is invertible, $r(f)$ is a coretraction and hence a strong monomorphism, whence its pullback $u$ in (5) is a strong monomorphism. Since $k$ is a strong monomorphism in (6) and $e$ in (6) is invertible because $f \in M$, the composite $f=u k e$ is a strong monomorphism.

\section{Small suprema of reflective subcategories}

Consider reflective subcategories $C_{i}$ of a strongly-finitelycomplete $A$ and the reflective factorization systems $\left|E_{i}, M_{i}\right|$ that correspond to them by Theorem 12. If there is a factorization system $(E, M)$ where $E=\cap E_{i}$, it is clearly reflective by $(3)$, and it is 
obviously the supremum of the $\left(E_{i}, M_{i}\right)$ in the set of all factorization systems, and a fortiori in the set of reflective factorization systems. Accordingly the corresponding $C=M / 1$ is the supremum of the $C_{i}$ in $\operatorname{Ref} A$. The following is an analogue of Theorem 3.1 of Borceux and Kelly [2], who dealt with the sirmpler case of small suprema in the ordered set Loc $A$ of localizations of A :

THEOREM 14. Let A be strongly complete [respectively strongly finitely complete] and let $\left\{C_{i}\right\}_{i \in I}$ be a set of reflective subcategories with I small [respectively finite]. Set $E=\cap E_{i}$, and let $M$ be the smallest skein containing $\cup M_{i}$ which is stable under small [respectively finite] fibred products and all intersections of strong monomorphisms. Then $(E, M L$ is a reflective factorization system, so that the corresponding $C=M / 1$ is the supremom of the $C_{i}$ in Ref $A$.

Proof. $E$ is a skein since each $E_{i}$ is so, and $M$ is a skein by definition. Since $M_{i}=E_{i}^{\downarrow} \subset E^{\downarrow}$, and since $E^{\psi}$ is a skein stable under fibred products by the remarks preceding (1), we have $M \subset E^{\dagger}$. It remains to show that any $f: A \rightarrow B$ has an $\left[E, M \mid\right.$ factorization. Let $f=m_{i} e_{i}$ be its $\left(E_{i}, M_{i}\right)$ factorization, and form the fibred product

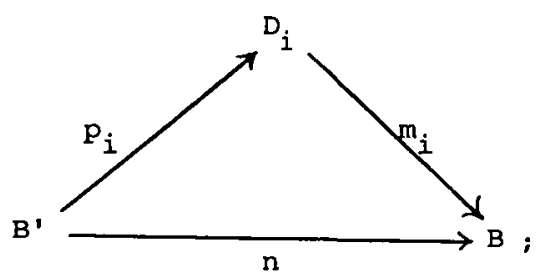

then $f=n f^{\prime}$, where $f^{\prime}: A+B^{\prime}$ is the unique map satisfying $p_{i} f^{\prime}=e_{i}$ for all $i$. Factorize $f^{\prime}$ as

$$
A \underset{f^{\prime}}{\longrightarrow} B^{\prime} \quad=\quad A \underset{e}{\longrightarrow} E \underset{k}{\longrightarrow} B^{\prime},
$$

where $k$ is the intersection of all those strong subobjects of $B^{\prime}$, lying in $M$, through which $f^{\prime}$ factorizes. By the definition of $M$, we have $n \in M$ and $k \in M$; it remains to show that $e \in E$, or 
equivalently that $e \in E_{i}$ for each $i$.

Let $e=m_{i} e_{i}^{\prime}$ be its $\left(E_{i}, M_{i}\right)$ factorization. since

$$
e_{i}=p_{i} f^{\prime}=p_{i} k e=p_{i} k m_{i}^{\prime} e_{i}^{\prime}
$$

and since $e_{i}$ and $e_{i}^{!}$belong to $E_{i}$, we have $p_{i} k m_{i}^{\prime} \in E_{i}$ by (2). Because $m_{i} ! \in M_{i}$, it follows from Proposition 13 that $m_{i}^{\prime}$ is a strong monomorphism. Since $e$ factorizes through the strong monomorphism $m_{i}^{\prime}$ lying in $M$, it follows from the definition of $k$ that $m_{i}$ is invertible. Thus $e \in E_{i}$ for each $i$, and hence $e \in E$.

The following, in its statement and in its proof, is a modification of the result given for localizations in Theorem 3.3 of Borceux and Kelly [2]:

THEOREM 15. In the situation of Theorem 14, $C$ is the strong-limit closure [respectively strong-finite-limit closure] in $A$ of $U C_{i}$.

Proof. Write $D$ for the closure in question of $\cup \mathcal{C}_{i}$; clearly $D \subset C$ since the reflective $C$ is closed in $A$ under all limits that exist; and it remains to show the converse.

Define as follows a set $N$ of maps in $A:$ the map $f: A \rightarrow B$ lies in $N$ if, for every pullback

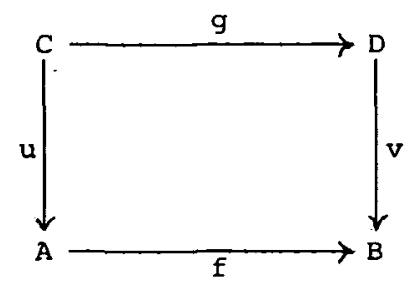

with $D \in D$, we have $C \in D$.

Since the pullback along $f h$ is the pullback along $h$ of the pullback along $f$, it is clear that $N$ is a skein. Since the pullback along $w v$ is the pullback along $v$ of the pullback along $w$, it is clear 
that $N$ is stable under pullbacks. Moreover $N$ is stable under such fibred products as exist, and hence under small [respectively finite] ones and all intersections of strong monomorphisms. To see this, consider a diagram

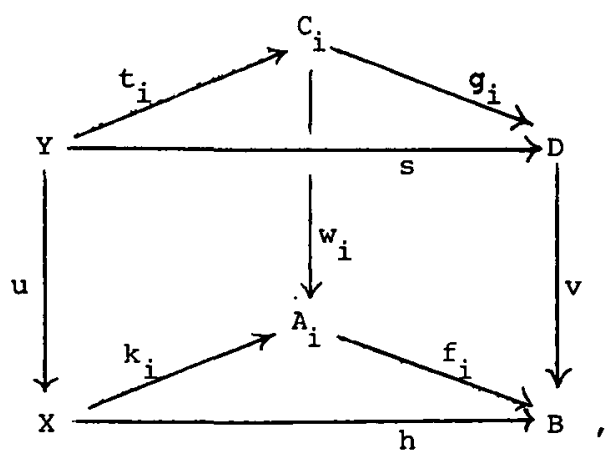

where the base of the prism is a fibred product, and where $u, s$ constitute the pullback of $v$ and $h$. If we define $w_{i}$ and $g_{i}$ as the pullback of $v$ and $f_{i}$, there are unique $t_{i}$ that complete the commutative diagram. Since pullback along $v$ is a right-adjoint functor $A / B \rightarrow A / D$, the top of the prism is again a fibred product. Now if each $f_{i} \in N$ and $D \in D$, we have each $C_{i} \in D$, whence $Y \in D$ because $D$ is closed under small [respectively finite] limits, so that $h \in N$.

Since $C_{i} \subset D$ and $D$ is closed under finite limits, it is clear that mor $C_{i} \subset N$. Because $N$ is stable under pullbacks and fibred products, it follows from Proposition 10 that each $M_{i} \subset N$; and now Theorem 14 gives $M \subset N$.

If $A \in C$, the unique map $f: A \rightarrow 1$ is in $M$ by Proposition 10 (since of course $1 \in C$ ) and is hence in $N$. Applying (9) with $v$ the identity map of 1 , and recalling that $1 \in D$, we have $A \in D$. Thus $C \subset D$, as required. 
Remark 16. We have seen in Remark. 8 above that large suprema need not exist in $\operatorname{Ref} A$ for a strongly-complete $A$, even under extra hypotheses on $A$. We now observe that, even when a large supremura $C=V C_{i}$ does exist, it need not be the strong-limit closure of $U C_{i}$. The counter-example is contained in Example 3.7 of [2]. We take for A the ordinal sum $1+\infty$, the dual of the ordered set $\infty+1$ consisting of all ordinals $\leq \infty$. This $A$ is locally small and admits all limits and colimits, even large ones; it is trivially weakly wellpowered and weakly cowellpowered, although neither wellpowered nor cowellpowered; and it trivially admits a generator and a cogenerator. The reflective subcategories $C_{B}=\left[\beta^{O P}, O^{O P}\right]$ for $\beta \in \infty$, where $\beta^{O P}$ denotes the ordinal $B<\infty$ as an element of $\infty O P$, have as their supremum $A$ itself; but $\cup C_{B}=\infty O P \subset A$ is already closed in $A$ under small limits and all intersections of strong monomorphisms.

\section{References}

[1] J. Adámek and J. Rosický, "Intersections of reflective subcategories", (in preparation).

[2] F. Borceux and G.M. Kelly, "On locales of localizations", J. Pure App Z. AZgebra, 46 (1987), (to appear).

[3] R. Börger, W. Tholen, M.B. Wischnewsky and H. Wolff, "Compact and hypercomplete categories", J. Pure Appl. Algebra 21 (1981), 129-144.

[4] C. Cassidy, M. Hébert and G.M. Kelly, "Reflective subcategories, localizations and factorization systems", J. Austral. Math. Soc. Ser. A. 38 (1985), 287-329; Corrigenda Ibid 41 (1986), 286.

[5] P.J. Freyd and G.M. Kelly, "Categories of continuous functors, I", J. Pure Appl. AZgebra 2 (1972), 169-191.

[6] G.M. Im and G.M. Kelly, "Some remarks on conservative functors with left adjoints", J. Korean Math. Soc. 23 (1986), 19-33.

[7] G.M. Kelly, "Monomorphisms, epimorphisms, and pull-backs", J. Austral. Math. Soc. 9 (1969), 124-142. 
Pure Mathematics Department, University of sydney, New South Wales, 2006 Australia. 